\title{
Murray Valley encephalitis virus
}

\author{
Ingrid A. Evans ${ }^{\mathrm{A}}$, Linda Hueston ${ }^{\mathrm{B}}$ \\ and Stephen L. Doggett ${ }^{\mathrm{C}}$ \\ A NSW Public Health Officer Training Program, \\ NSW Department of Health \\ ${ }^{\mathrm{B}}$ Arbovirus and Emerging Diseases Unit, Institute of Clinical \\ Pathology and Medical Research, Westmead Hospital \\ ${ }^{\mathrm{C}}$ Department of Medical Entomology, Institute of Clinical \\ Pathology and Medical Research, Westmead Hospital
}

Murray Valley encephalitis virus (MVEV) is a mosquitoborne flavivirus closely related to the Japanese encephalitis and West Nile viruses. ${ }^{1}$ The vast majority of infections are asymptomatic or mild; however, the most severe form of disease - encephalitis - has a mortality rate of up to $30 \%$, with $30-40 \%$ of survivors having permanent neurological disabilities. ${ }^{2}$ There is no specific treatment or vaccine for the disease. MVEV is endemic in northern Australia $^{3}$ but has rarely been seen in south-eastern Australia since a national epidemic in 1974 of 58 cases with $20 \%$ mortality. ${ }^{4}$

The primary vector of MVEV during epidemics is thought to be the common banded mosquito, Culex annulirostris, which prefers breeding in shallow, warm, fresh water. ${ }^{5}$ The primary vertebrate hosts of MVEV are thought to be water birds, which act as reservoirs for the virus. The Nankeen (Rufous) Night Heron, cormorants and the Australian Darter are considered important reservoirs, but many bird species can be infected with MVEV. ${ }^{6}$

Much remains unknown about MVEV ecology. There are two main theories explaining the appearance of the virus in south-eastern Australia:

1) MVEV is constantly present in small, unknown locations from which it amplifies during periods of drought through the highly localised accumulation of vectors or following heavy rainfall (widespread multiplication of mosquitoes and birds); ${ }^{7,8}$ and/or

2) MVEV is introduced via infected birds from endemic areas in northern Australia after heavy rainfall in the central and eastern parts of the continent. ${ }^{9}$

A new third theory suggests that atmospheric conditions, in particular low pressure cells, may support the spread of the virus. ${ }^{10}$

\section{MVEV surveillance systems}

MVEV activity is monitored in susceptible regions of New South Wales (NSW) by detecting MVEV in trapped mosquito samples and/or detecting seroconversion to MVEV in sentinel chicken flocks. These surveillance systems are intended to provide an early warning of MVEV activity. Monitoring of meteorological events can also give a broad indication of likely vector and host activity, while climatic indices can be used in epidemic predictive modelling. ${ }^{411-13}$

Most recently, in 2001 a small serosurvey in the Macquarie Marshes in north-western NSW found evidence of MVEV activity in humans post-1974 and pre-2000-2001. MVEV seroconversion was detected in sentinel chickens in northern inland NSW in 2000-2001, and again at Menindee in 2003, with no human cases. In 2008, both mosquito and chicken samples indicated MVEV activity in central NSW and the Riverina, with one mild non-encephalitic human case detected in the Macquarie Marshes. ${ }^{13}$

The question remains: why is MVEV disease not seen more often in south-eastern Australia despite the recent evidence for virus activity? MVEV has a high sub-clinical infection rate: it has been estimated that only one in 800-1000 infections result in clinical disease. ${ }^{14}$ This combined with a low index of suspicion amongst general practitioners means that mild cases are likely to be missed (the human case in 2008 had insisted on being tested for MVEV).

Since 1974, rainfall, land use, water and wetland management in susceptible regions have changed and are likely to have had an impact on the ecology of the virus. Sentinel chicken flocks and mosquito trapping sites have also been reduced, leading to less opportunity for detection of the virus.

\section{Public health response}

The detection of MVEV via the surveillance program has prompted a rapid release of public health warnings with personal protection against mosquito bites being encouraged. The timely release of these health messages has hopefully encouraged behavioural changes so that exposure to potentially viraemic mosquitoes has been reduced. These warnings, which can be targeted to the lifestyles and literacy levels of at-risk communities, also aim to increase awareness of possible symptoms for both the general public and relevant health professionals. Raising the index of suspicion for MVEV amongst local health professionals is vital for early notification of suspected cases to public health units and for the collection of pathology samples for testing at the Institute of Clinical Pathology and Medical Research at Westmead Hospital. 


\section{Conclusion}

MVEV should be considered as a possible - albeit rare cause of disease in rural NSW. The high mortality and morbidity associated with MVEV can invoke community fear, media alarm and public health concern. This justifies monitoring and preparation for the unpredictable return of MVEV disease to south-eastern Australia.

\section{References}

1. Mancini EJ, Assenberg R, Verma A, Walter TS, Tuma R, Grimes JM et al. Structure of the Murray Valley encephalitis virus RNA helicase at 1.9 Angstrom resolution. Protein Sci 2007; 16(10): 2294-300. doi:10.1110/PS.072843107

2. Cordova SP, Smith DW, Broom AK, Lindsay MD, Dowse GK, Beers MY. Murray Valley encephalitis in Western Australia in 2000, with evidence of southerly spread. Commun Dis Intell 2000; 24(12): 368-72.

3. Broom A, Sturrock K, van Heuzen B, Lindsay M, Smith D. Seroconversions in sentinel chickens provide an early warning of Murray Valley encephalitis virus activity in Western Australia. Arbo Res Aust 2001; 8: 43-7.

4. Forbes JA. Murray Valley encephalitis 1974: also the epidemic variance since 1914 and predisposing rainfall patterns. Sydney: Australasian Medical Publishing Company; 1978.

5. Spencer JD, Azoulas J, Broom AK, Buick TD, Currie B, Daniels PW et al. Murray Valley encephalitis virus surveillance and control initiatives in Australia. National Arbovirus Advisory Committee of the Communicable Diseases Network Australia. Commun Dis Intell 2001; 25(2): 33-47.

6. Anderson SG, Donnelly M, Stevenson WJ, Caldwell NJ, Eagle M. Murray-Valley encephalitis: surveys of human and animal sera. Med J Aust 1952; 1(4): 110-4.
7. Miles JA, Howes DW. Observations on virus encephalitis in South Australia. Med J Aust 1953; 1(1): 7-12.

8. Anderson SG, Eagle M. Murray Valley encephalitis; the contrasting epidemiological picture in 1951 and 1952. Med J Aust 1953; 1(14): 478-81.

9. Marshall ID, Woodroofe GM, Hirsch S. Viruses recovered from mosquitoes and wildlife serum collected in the Murray Valley of South-eastern Australia, February 1974, during an epidemic of encephalitis. Aust J Exp Biol Med Sci 1982; 60(5): 457-70. doi:10.1038/icb.1982.51

10. Finlaison DS, Read AJ, Kirkland PD. The New South Wales bovine ephemeral fever outbreak 2008 - evidence for windborne transmission (Abstract). 10th Arbovirus Research in Australia Symposium, Coffs Harbour, NSW, 2008. p. 74.

11. Nicholls N. A method for predicting Murray Valley encephalitis in southeast Australia using the Southern Oscillation. Aust J Exp Biol Med Sci 1986; 64(6): 587-94. doi:10.1038/icb.1986.62

12. Bennett NM. Murray Valley encephalitis. Commun Dis Intell 2001; 25(3): 154-5.

13. The New South Wales Arbovirus Surveillance and Mosquito Monitoring Program website. Mosquito/Virus Results. Available at http://medent.usyd.edu.au/arbovirus/results/ results.htm (Cited August 2009.)

14. Broom A, McKinnon M, Miller M, Moran R, Russell R, Whelan P et al. Interim National Guidelines for the Prevention, Management and Control of Murray Valley Encephalitis Virus. Communicable Diseases Network Australia, 2005. 